\title{
An Evaluation Tool for Research of User Behavior in a Realistic Mobile Environment
}

Ivo Maly, Zdenek Mikovec, Jan Vystrcil, Jakub Franc, Pavel Slavik

Faculty of Electrical Engineering, Czech Technical University in Prague

Tel: +420 224357655

Fax: +420224357556

\{malyi1,xmikovec, vystrjan, francjak, slavik\}@fel.cvut.cz

Abstract: User behavior is significantly influenced by the surrounding environment. Especially complex and dynamically changing environments (like mobile environment) are represented by a wide variety of extraneous variables, which influence the user behavior in an unpredictable and mostly uncontrolled way. For researchers it is challenging to measure and analyze the user behavior in such environments. We introduce a complex tool - the IVE tool - which provides a unique way of context visualization and synchronization of measured data of various kinds. Thanks to this tool it is possible to efficiently evaluate data acquired during complex usability tests in a mobile environment. The functionality of this tool is demonstrated on the use-case "Navigation of visually impaired users in the building with support of a navigation system called NaviTerier". During the experiment we focused on collection and analysis of data that may show user stress and which may influence his/her ability to navigate. We analyzed objective data like Galvanic Skin Response parameter (GSR), Heart Rate Variability parameters (HRV) and audio video recordings and also subjective data like the user's subjective stress feeling and observation of the user's behavior.

Keywords: user behavior, context sensitivity, measuring usability, a11y, user experience

\section{Introduction}

The research of user behavior in mobile environments faces a problem with the trade-off between the ability to measure all the parameters in appropriate detail (possible mostly in lab environment only) and the ecological validity of the experiment (which can be ensured by field studies). Field studies performed in natural mobile environments (building, street, etc.) introduce two main problems. First, the measurement of needed behavioral parameters is rather difficult if not impossible in comparison to the lab environment; e.g. recording finger movement on the display or recording of the whole environment influencing directly the user behavior. Second, the complexity and dynamics of the natural environment with a wide variety of extraneous variables influence the user behavior in an unpredictable and mostly uncontrolled way.

On the one hand, we as researchers want to evoke this exact situation, which is ecologically valid and has potential to show us realistic user behavior in the 
mobile environment. On the other hand, there is a problem with the interpretation of such observed behavior as we cannot control all the variables of the study. The question is if we are able to measure a sufficient amount of data with sufficient precision and if we are able to analyze these data in such a way that we could correctly interpret the behavior observed.

In this paper we will introduce a complex tool - the IVE tool - for the visualization of multiple data sources (generated by various measurements of users behavior) and evaluation of participant behavior in a natural mobile environment. The benefit of unique context visualization plug-in and synchronization functionality during the evaluation process will be demonstrated by the use-case "Navigation of visually impaired users in the building with support of a navigation system called NaviTerier". This paper demonstrates efficient evaluation of data acquired by a complex usability test in the mobile environment by means of a special evaluation tool called IVE.

\section{Use-case: Navigation of visually impaired in the building}

The use-case we have chosen to demonstrate the analytical IVE tool and the process of evaluation and interpretation of user behavior observed in the mobile environment is "Navigation of visually impaired person inside buildings". The visually impaired person is walking through the building from his/her actual position (for example the entrance) to the requested destination with help of the navigation system called NaviTerier. NaviTerier is a mobile interior navigation system for visually impaired users running on a standard mobile phone typically used by visually impaired persons. The main principle of the system is based on well prepared descriptions of the route in the interior suited to the needs of visually impaired users. The NaviTerier application is utilizing standard Text To Speech application installed on the user's cell phone. A description of the complete route is divided into logical segments that are reproduced step-by-step to the user as he/she is moving through the route (see Fig 1 ).

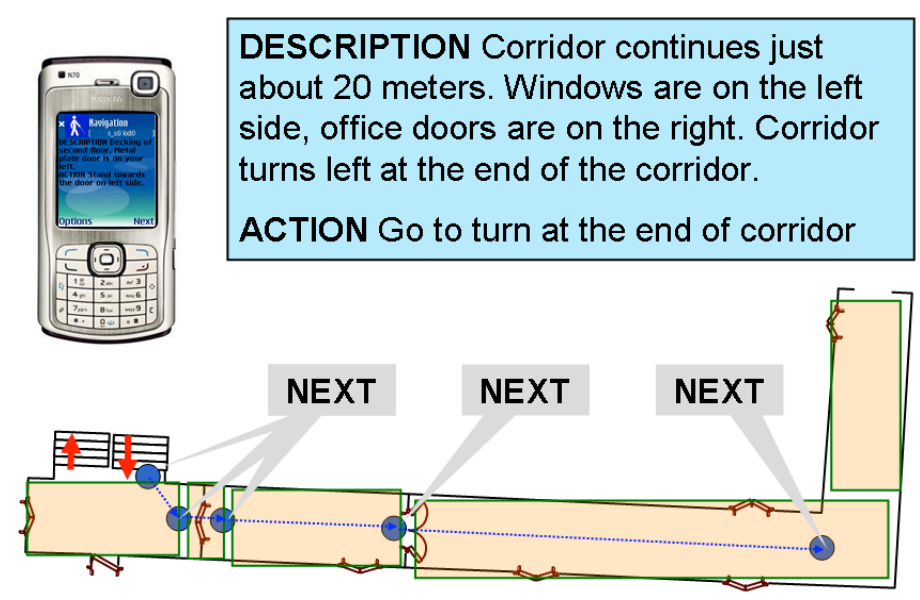

Fig 1 NaviTerier - navigation principle. 


\section{Early stages of research}

The main research issue, besides the usability of the system control and understandability of the navigation description, was the question: What are the most important orientation cues that should be presented to the visually impaired user to ensure reliable and efficient navigation and orientation in an unknown area? The ideal way to answer this research question is to perform a usability study in a real environment. The task should be navigation to some destination in a building. After the walkthrough the participants should be asked about the importance of the orientation cues on the route.

We have performed two qualitative studies to evaluate usability of control of our navigating system and also to improve descriptions of the environment to be valuable and easily understandable for the users. To analyze the importance of features (objects, shapes of the corridors, etc.) on the route and indicate the candidates for important orientation cues we have also performed post-test interviews to get feedback from participants. We have asked the participants to try to recall the whole route they have passed in order to check our assumption that the most important features will be remembered much better than the less important ones.

Surprisingly we have observed that the ease of remembering the features on the route is dependent not only on the type of the feature, but also on their position on the route. In particular we have observed that there were parts of the route where the participants were not able to recall features (like doors, shape of the corridor) they were correctly reporting in the rest of the route. We have made an assumption that this situation could be caused by a stress reaction, which negatively influences the cognitive processes, especially attention and memory performance in its acquisition phase [1].

\section{Validity issues}

There is a whole range of techniques for studying spatial environmental knowledge and its acquisition both by sighted and visually impaired persons. Most popular are virtual (for example Foo et al. [13], Gillner S, Mallot HA [19], Kjeldskov [6]) or micro-scale artificial environment settings (for example Tellevik [20] or Ochaíta and Huertas [21]) that allow researchers to control the environment settings and parameters to a great extent. When studies are conducted in real environments they mostly focus on short routes rather on complex real world like situations. Studying spatial knowledge acquisition in real world large-scale complex environments is very rare. Such an approach brings numerous methodological challenges as researchers do not control the environment setting and parameters so well.

Kitchin, Jacobson [9] state that the wayfinding in large-scale real-world spaces is different from wayfinding in limited areas. Natural large-scale environments provide different sources of environmental cues and a dramatically different amount of environmental information than the artificial limited settings. They also 
show distractions based on situational context (other people, obstacles, diversions along the route). Studies conducted in artificial and small scale environments suffer from the lack of ecological validity that prevents the research findings to be extrapolated into wayfinding and spatial knowledge acquisition in the daily lives of the visually impaired [9].

Our research approach follows Jacobson's and Kitchin's call [9] to 'start to assess the knowledge and abilities within complex real-world environments that everyone inhabits, rather than inferring that results from the laboratory will exist in natural settings'. This approach will require the collection of much larger amounts of data of various natures that should be efficiently evaluated.

\section{Experiment}

In this section we will describe an experiment, which will serve as a demonstration of problems that the researcher has to face when observing user behavior in a realistic mobile environment. We will define research topics and requirements on the measurement methods we need to use to gather appropriate data for evaluation.

\subsection{Research topics}

Based on the previous research we have formulated the following research topics:

1. Is it true that the acute stress reaction following the stress stimuli influences negatively the ability to remember the features on the route?

2. Which features and to what extent are they affected by the decreased ability to remember caused by the stress reaction?

To investigate these topics we needed to setup an experiment where we will be able to generate stress stimuli, check if there will be invoked a stress reaction of the participant and to get information about the features remembered by the participant. The experiment setup had to calculate and to perform the test with two groups - an experimental and a control group. In the following sections we will describe the measuring methods used, the test route preparation and the experiment procedure.

\subsection{Measuring methods}

\section{Measuring stress}

We have studied several approaches for stress measurement to find those which will accommodate the following criteria:

- Non-invasive

- Continuous logging of potential stress level

- Resistant to direct influence of physical activity

- Suitable for mobile setup

Common methods based on measuring of urinary excretion rates of noradrenaline, adrenaline or salivary cortisol levels [14] were not suitable for our purposes because of the necessity of continuous detection. Analysis of pupil 
diameter to detect stress is not relevant due to target group of visually impaired users [15].

The most suitable methods to satisfy the above mentioned requirements appear to be the measurement of Galvanic Skin Response parameters (GSR) and Heart Rate Variability parameters (HRV).

\section{GSR Analysis}

Analysis using GSR is a method based on measuring of skin conductance that is rapidly changing in dependence on physiological changes caused by stress [11]. Stress activates the sympathetic nervous system, thus resulting in increased levels of sweat in the sweat glands and consequently increases the electric conductance of the skin [2]. Our experiment route (see section 5.3) was designed in a way that the stress stimuli are the only variables in the experiment and we expect that the GSR values will be primarily influenced by these stimuli. Appearance of other emotions that could influence GSR was checked using other data sources like subjective evaluation and observation of audio/video recording. In the experiment, we have used the GSR sensor BodyMedia SenseWear PRO ${ }^{1}$ in the form of a band that is placed on the back part of the upper arm. Data are logged to internal memory of the sensor. It also measures body temperature, accelerations, etc.

\section{HRV Analysis}

HRV analysis is a method based on ECG signal analysis, where specific changes in heart rate reflect physiological changes caused by stress [16], [17]. According to [2] HRV represents the variations in the beat-to-beat intervals. HRV analysis is prevalently used to assess the effect of autonomic regulation on the heart rate. It provides a dynamic nature of the interplay between the sympathetic and parasympathetic branches. The relation between the sympathetic and parasympathetic branch can be described by the ratio of low and high frequencies $L F / \mathrm{HF}^{2}$ in frequency spectrum obtained from beat-to-beat intervals in time. These frequencies are obtained by means of FFT (Fast Fourier Transformation). There is a balance between the sympathetic and parasympathetic branch under normal situations, placing the body in a state of homeostasis. However, under a state of mental stress, this balance will be altered. These states can be observed as differences between the values of $\mathrm{LF} / \mathrm{HF}$ ratio in time. Each value (LF/HF ratio) we calculate is based on analysis of certain interval of signal (sliding window). We have been working with a 4 minute long ${ }^{3}$ sliding window to get insight on how LF/HF HRV parameters change in time. In the experiment, we have used Kubios's HRV [5] application to calculate LF/HF ratio parameters (see Fig 2 for highlighted one 4 minute window).

1 http://sensewear.bodymedia.com

2 According to [10] LF/HF parametr of $\mathrm{HRV}, \mathrm{LF}=0,04-0,15 \mathrm{~Hz}, \mathrm{HF}=0,15-0,4 \mathrm{~Hz}$

$3 \quad$ Bayevski [10] uses 5 minute long sliding window. As we have relatively short measurements of signal, we have used 4 minute sliding window to gain more samples of LF/HF ratio 


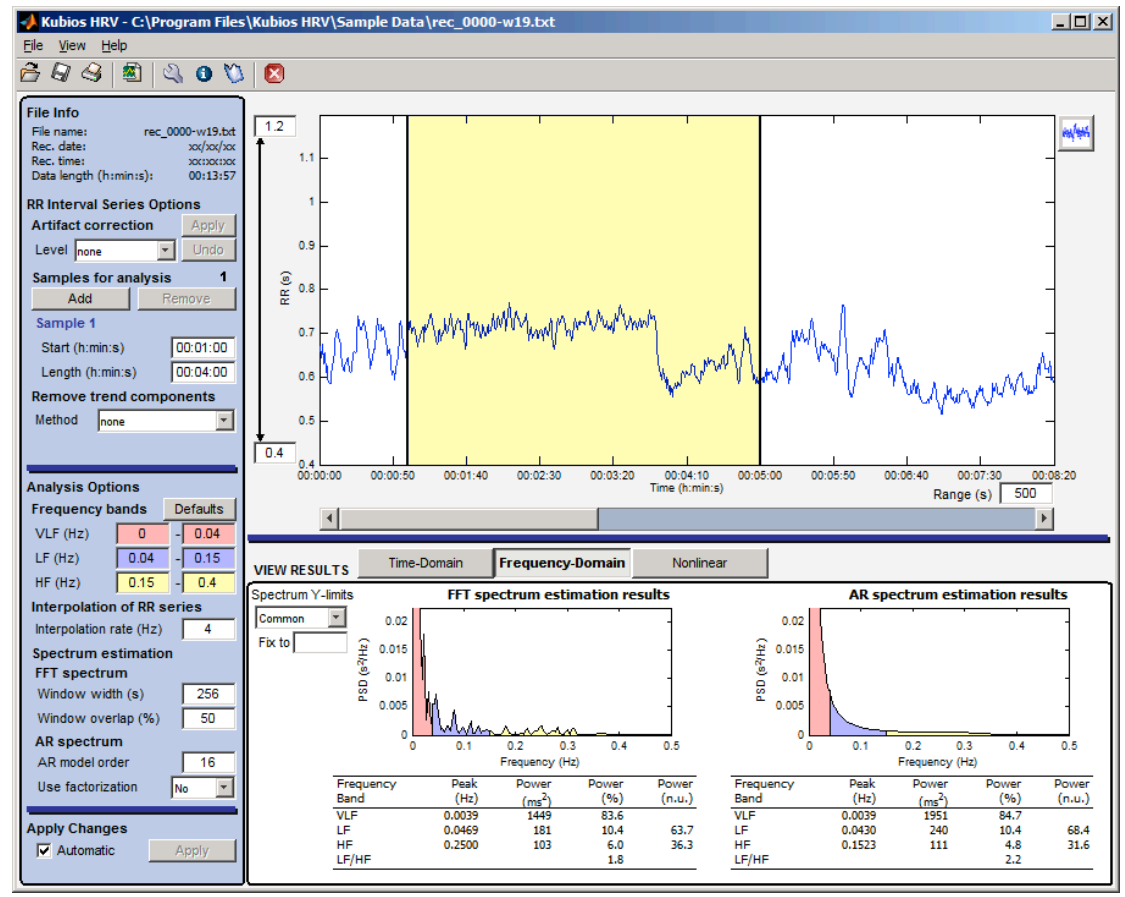

Fig 2 Kubios HRV with 4 minutes sliding window

We have also used an ECG sensor in the form of a chest strap ${ }^{4}$ that was installed on the participant's body and a wire connected logging unit has been placed in a small backpack carried by participants [18].

\section{Subjective evaluation}

During the post-test phase the subjective feeling of the stress was evaluated. The route, through which the user was walking, has been divided into 6 parts that were described to participants by the evaluator. Participants were invited to sort them in descending order according to their subjective feeling of stress in those parts. The force choice technique (each segment had to be ordered) was used. The forced choice principle was used to identify the most stressful segment for later comparison with other data sources.

\section{Camera}

In order to record the exact movement of the participant a small camera has been attached to a shoulder strap of the backpack. The camera recorded the area in front of the participant. Participants were also recorded by a third person for possible better analysis of the context of participant behavior.

\section{Observation in the field}

Physical interaction with all objects on the route was registered in the log sheet. These data were gathered for further comparison with the objects mentioned by the participant in the interview during the post-test phase. 


\section{Logging}

The NaviTerier application is logging all interactions the user has performed with the mobile device so we do not need any direct screen capturing of the display.

\section{Post-test interview}

After completion of the test route, participants were asked to freely describe the route verbally to gain insight into how they remember the route they have taken. All remembered objects had to be mentioned and were registered in the log sheet.

\subsection{Test route preparation}

Our plan was to prepare a route identical for both groups of users (experimental and control). The difference would be in one segment only, which would contain real stress stimuli for the experimental group and no stress stimuli for the control group. All other segments had to be the same. Unfortunately, we were not able to find a route that would comply with these requirements. But according to Gray, J. A. (1987), we can simulate the stress environment by verbal and physical stimuli with a similar effect as in a real stress environment [12]. Therefore we decided to force evocation of stress in one of the segments for the experimental group of participants and all other parts of the route remained unchanged. The second group of participants was treated as the control group.

Forced evocation of stress has been performed through realistic auditory and haptic stimuli associated with a potentially threatening environment. In one of the corridors reconstruction work has been simulated. Participants have been warned by the NaviTerier of potential appearance of dangerous obstacles. The floor was covered with plastic foil and a vertical barrier from the plastic foil was installed across the corridor. Buckets and other instruments were installed on the floor to ensure a more realistic sense of reconstruction. The control group met only the standard corridor without any obstacles in the mentioned corridor.

\section{Placing stressful stimuli}

Expecting individually different reactions to the stress stimuli, we have chosen to measure the level of stress objectively through GSR and HRV methods as a verification mechanism.

Placing stressful stimuli in one of the segments of the route did not serve as a direct precursor of stress reaction. When initially analyzing both HRV data and subjective reports of the participants we have found that the unsystematic and uncontrolled situational variables on the route (e.g. random social encounters, environmental features such as distant noises that could work as navigation cues) were causing significant stress levels in both experimental and control groups. 


\subsection{Test procedure}

In the experiment we were observing two groups of users. In experimental group we observed 12 blind participants and in the control group we observed 10 blind participants. The complete experiment consisted of 5 consequent phases (pretest phase, training phase, break, test phase, post-test evaluation).

\section{a) Pre-test phase}

During the pre-test phase participants were acquainted with the study procedure. The principle of the NaviTerier navigation system was explained and instructions on how to operate a cell phone with the NaviTerier application were given to the participants. All sensors described in section 5.2 (ECG, GSR, camera) were installed on the participant's body.

\section{b) Training phase}

During the training phase participants went through a training route that was totally different from the test route. The training phase took approximately 10 minutes and participants were followed by test conductors to help with any problems that might occur. The main purpose of the training phase was to get participants familiar with the navigation device and to avoid any technical problems during the test phase. Only the arm placed GSR sensor was turned on in this phase as it needs some time to "warm up" for correct measurement. After passing the training route, participants were led back to the lab to have a rest, settle down and to be instructed for the test phase.

The ECG measurement system and audio-video recording had been switched on. Participants were informed that they will go through the test route independently and also given important instructions to try to remember the route precisely so next time they could go through the route on their own.

\section{c) Test}

The whole test route consisted of 15 segments including stairways, corridors and doors (see Fig 3). Participants were led at the start of the first segment that was a square-shaped stairway. It had to be stepped 2 floors down. To avoid any potential problems with opening of doors, all doors on the route were kept opened. 


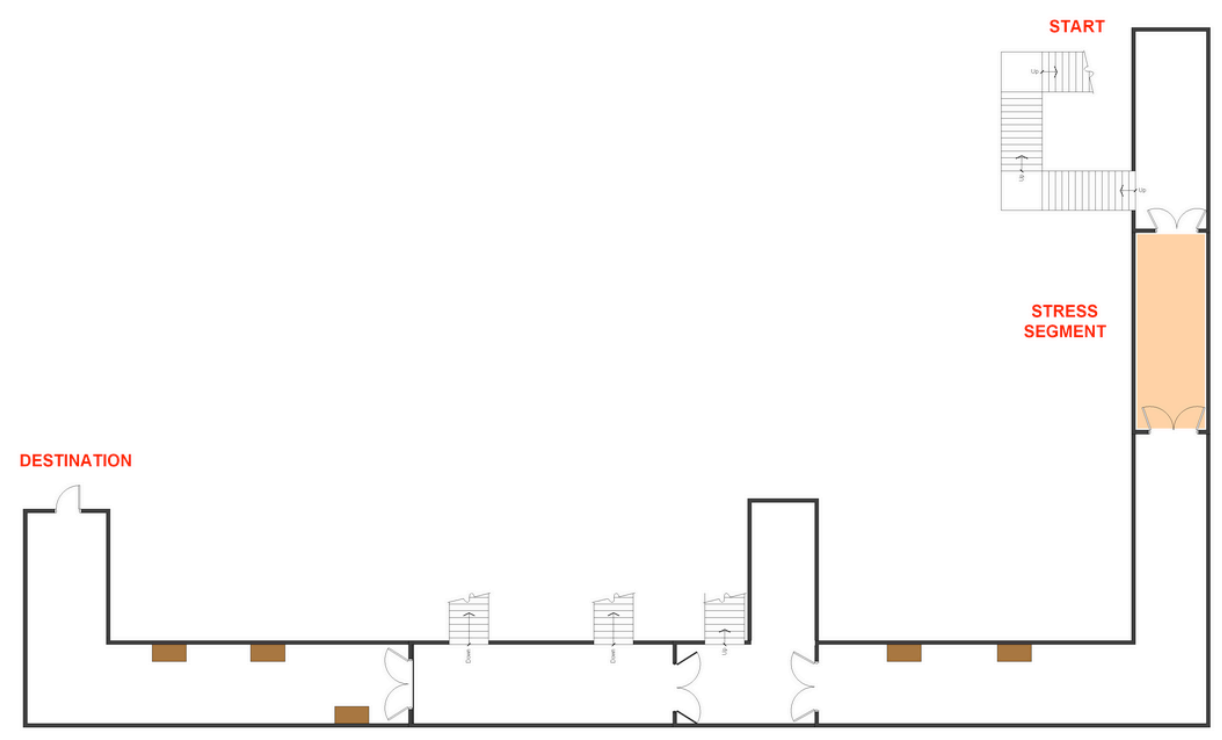

Fig 3 Test route schema

After passing the first doors, there was a so called "stress segment" highlighted in Fig 3. Depending on whether the participant was in the experimental or control group, there was either the setup as described in section 5.3 or just a normal corridor. The rest of the route consisted of corridors, turns and doors.

To avoid any potential disruption of the test there was special emphasis on carrying out all observations (third person audiovisual recording and field observation of participant interaction with the environment) without being noticed by the participants.

\section{d) Post-test evaluation}

Participants were returned to the lab and all sensors were removed from their body. They were asked to describe the route verbally to gain the information about how they remember the route features they have passed. All remembered features (objects, corridors, crossings, etc.) had to be mentioned and were registered in the log sheet.

Six parts of the route (defined in section 5.2) were described to participants. Participants were than invited to sort these parts in descending order according to their subjective feeling of stress in those parts. Forced selection principle had been used so no two parts could be marked with the same stress level.

Participants were informed that a video of their walk through had been taken by a third person and they were asked for permission for using these video recordings for further analysis. 


\section{Analysis and scientific evaluation of measurements}

In order to perform scientific evaluation which is typically determined by research topics we need tools, which will allow us to perform efficient human based observation and analysis of measured values. The analysis is typically a process, where we are trying to find interesting patterns and correlations in the data. The analysis could be facilitated by the ability to define combinations of various parameters and compare this newly derived information with other previously measured data.

This scientific evaluation process must be supported by complex visualization because it involves analysis of a wide range and large amount of data available to the researcher. This is additional to the data measured by the experimental setup also providing contextual data (like state of the NaviTerier application, environment surrounding the test participant), which are essential for interpretation of the observed behavior patterns. This data is typical of a very different nature and data types (e.g., logs, AV data, and questionnaires externalization + subjective stress, observation reports - incidence with objects on the route). The tool must be able to cope with the processing, visualization and synchronization of these data sources.

For scientific evaluation it is also essential to be able to inspect the visualized data from different points of view to search for interesting behavioral patterns. Finally it must be possible to analyze the data across several participants to support the analysis of similarities or differences in user behavior or in other collected data. We would like to see selected data side by side, with possibilities to align them or to highlight same situations, e.g. same tasks or similar stress data trends.

The analytical tool requirements can by summarized as follows:

- the length of necessarily observed video must be efficiently minimized

- only video is viewed sequentially, other data should be visible at a glance with possibility to search, directly access any part of data and focus on details

- data views must be synchronized

- wide range of data of very different types must be visualized

- annotation functionality must allow efficient derivation of new information from existing one

- any data source must be able to serve as master data, where the focus of the researcher is paid and other data must adapt synchronously

From the data source point of view, it is necessary that the analytic tool can work with the following data sources:

- application model of the tested application

- log files from tested application

- observation of interactions with objects (e.g. doors, flowers, windows) during the test 
- post-test analysis (e.g., subjective evaluation of stress level on the route, remembered objects the participant interacted with on the route)

- HRV data recorded during study and analyzed using Kubios HRV [5]

- GSR data and two audio/video recordings

We found out that most tools for visual data analysis offer only static visualization of generic spreadsheets or database data in a form of predefined visualizations, like plots, maps, graphs or dashboards. Some tools (e.g. VisiFire [7]) allow for animations of the data changes. Generally, none of these tools allow synchronous visualization of the spreadsheets or database data together with multimedia files, as in. video recordings. Observer XT tools from Noldus [8] allow complex analysis of data from behavioral studies together with multiple video recordings. However, the Observer XT is not able to provide us with custom visualizations of data like visualizations of application states in a form of segment view or show us non-standard temporal data (with no direct mapping of values to time line), like HRV values from Kubios, synchronized with video recording.

Because we have not found any suitable analytic tool we decided to use the IVE (Integrated Interactive Information Visualization Environment) tool and develop new plug-ins for data source import and data visualization that will suit our test. The tool was in detail presented in [3]. An advantage of the IVE tool, compared to generic visualization toolkits like VTK, is that it allows quick creation of custom data importers and custom visualization plug-ins synchronized with multimedia files. It is designed for analysis of temporal data and therefore it is easier to develop synchronized visualizations in it. Both the IVE tool and the plug-ins used and developed for the required data source visual analysis are described in the following sections.

\subsection{Description of IVE Tool structure}

IVE is an interactive tool for visual analysis of data from usability studies. IVE tries to give the usability expert the power of the qualitative analysis tool to understand user behavior but it also allows statistics calculation.

The IVE tool is based on the structure of Information Visualization Reference Model [4]. This model represents a framework suitable for implementation of various information visualization applications. A problem with tools for visual analysis is that in each study the data sources are in a different format and/or different types of data are available. The main advantage of the IVE tool is that it successfully copes with both problems using plug-ins.

In Fig 4 we can see how the process works. IVE uses an internal object database and converter plug-ins to convert data from raw sources into this database that basically contains records of key-pair or multimedia data. Because generic objects in the internal database would bring unnecessary complexity to both the conversion process and reusability of visualizations, we limit the type of internal data types. Currently the IVE works with:

audio/video recording,

- generic log file (list of records, each record contains list of key/value pairs), 
- task model,

- application model.

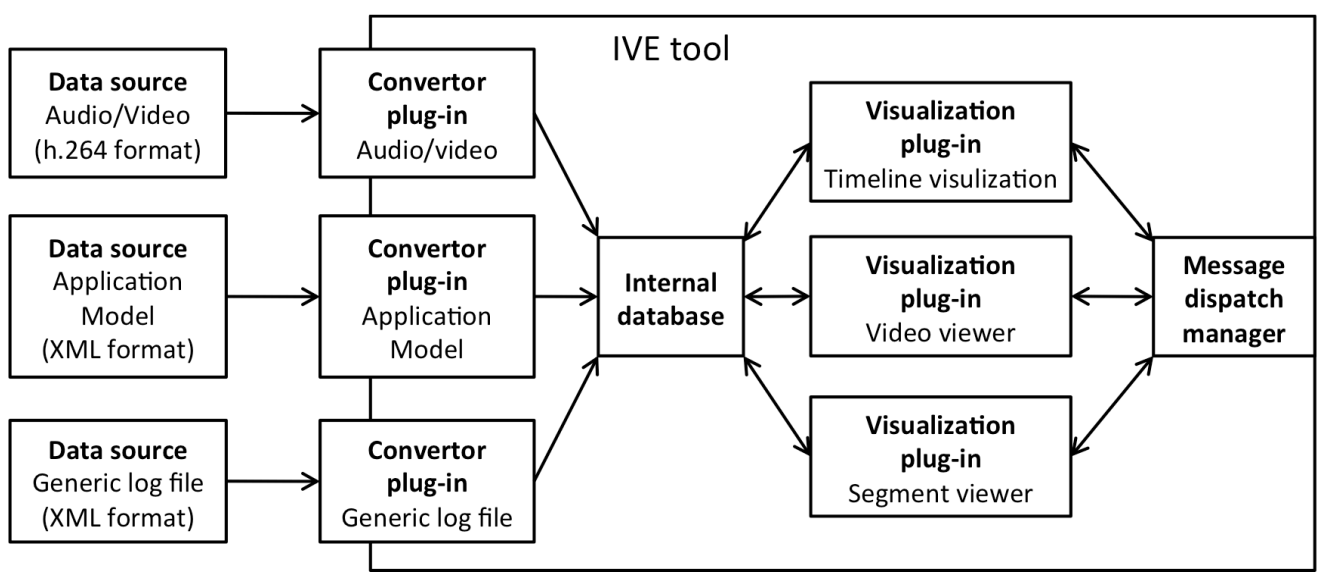

Fig 4 Schematic structure of the IVE tool and IVE convertor and visualization plug-ins.

Depending on the structure of available data, the usability expert can use a subset of visualization plug-ins or a subset of the visualization plug-in functionality. This means no data source is mandatory, but when more data sources are available, the IVE is able to take advantage of this fact and can allow interconnection of data and therefore enhancement of visualizations, e.g. interconnection of navigation segment visualization with navigation segment instructions for test participant and subjective stress rating of that navigation segment.

Because IVE limits the type of data stored in its internal database it is easier to develop new visualization plug-ins for IVE. Each visualization plug-in is developed as a plug-in with one or more views that have access to the IVE internal database and it can communicate with other plug-ins through a simple message dispatching system. The IVE tool with visualization plug-in views developed for analysis of data from NaviTerier navigation application is shown in Fig 5. Details of the plug-ins functionality and data visualization are in the following sections.

A visualization plug-in is instantiated using the IVE wizard that allows selection of the visualization plug-in and selection of data sets (log file, application model, audio/video recording) that will be used in the visualization plug-in views. Each visualization plug-in can announce which data sets are mandatory and it can also limit the amount of data sets. Some visualization plug-ins may announce changes to other visualization plug-ins, e.g. highlighting of the same information in other visualization plug-in views. Instead of storing this information in the internal database we used a central message dispatcher that collects and resends all messages between all visualization plug-ins.

The IVE tool was developed in Java using the NetBeans Platform and therefore it includes several features typical for this platform. First of all is the use of the builtin plug-in update manager, which is used for all plug-ins for the IVE tool and which allows for unified handling of plug-ins. Second, the tool uses a window 
management of the NetBeans platform that allows for a complex placement and manipulation or even undocking of the visualization plug-in views in the main panel of the tool.

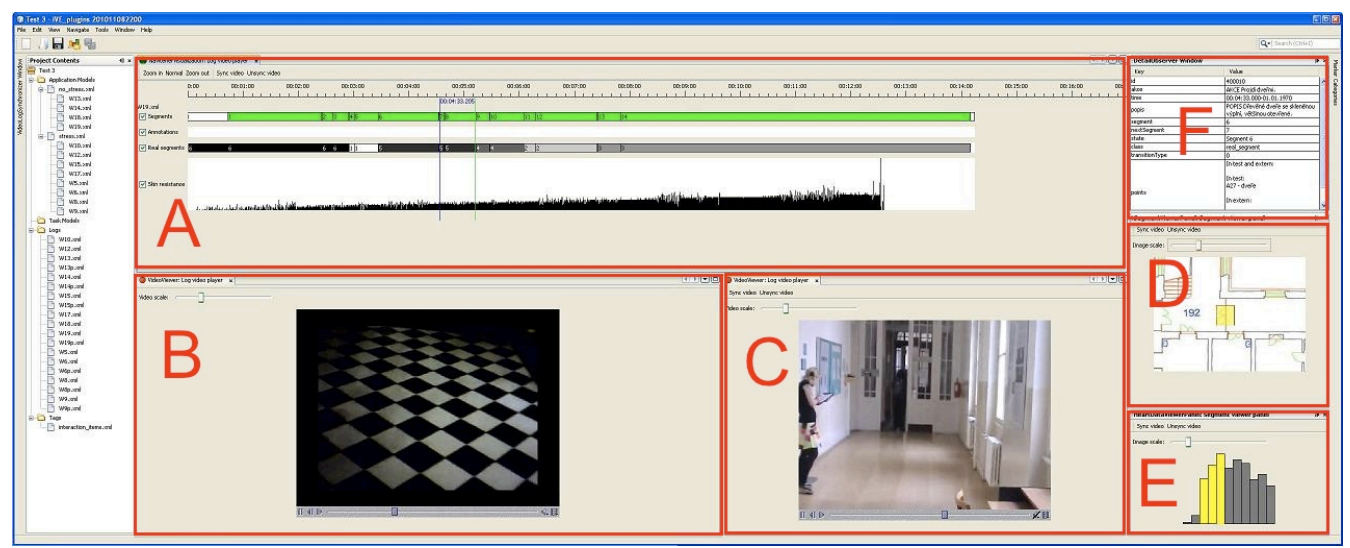

Fig 5 IVE tool with Timeline visualization plug-in (A), two Video viewer plug-ins (B, C) Segment viewer plug-in (D), HRV viewer plug-in (E), and Detail window $(F)$.

\subsection{Timeline Visualization Plug-in}

The timeline visualization plug-in is the plug-in that shows a combination of several data sources in one view (see Fig 6). This plug-in is able to show the log file of the application (NT Segments timeline), additional user annotations (Annotations timeline), the real position of the user in a segment (Real segments timeline) and GSR data (Skin conductance timeline). For each record in each line, there is the possibility to show details in the Detail window (described in following sections). Each timeline can be hidden with a checkbox in front of the timeline name, zoomed in and out using buttons on the tool bar and synchronized with the master video player. When the timeline is synchronized there is an indicator for the position in the video and current log file details are presented in the Detail window.

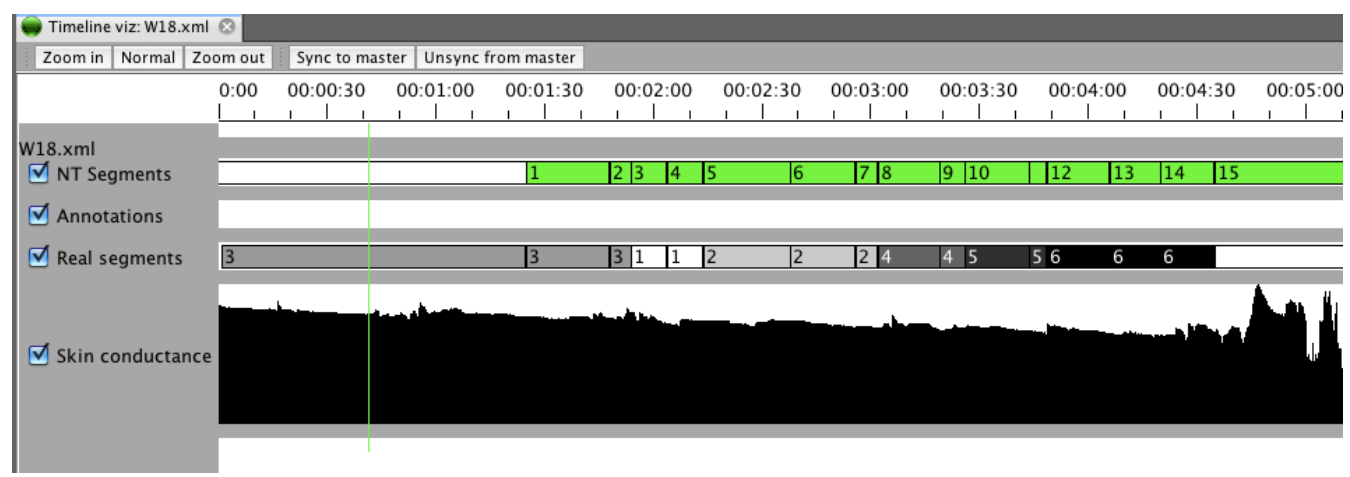

Fig 6 Timeline visualization

Each timeline uses a different visualization to present data. The NT Segments timeline shows rectangles that represent selection of new segments in the tested application. The color represents the type of action. e.g. green color (or ascending sequence of following rectangles) means that the user selected the next segment and he/she is probably following the test smoothly. Red color (or 
descending sequence of following rectangle labels) means that the user had to select the previous segment. That may signal that the user had problems and such a point should be further analyzed in the video.

The Real segments timeline shows the real position of the user in the segment. This position may differ from application segments when the user is lost or when he/she starts the next segment during his/her way to the next segment. The hue and the label represent the value of the subjective stress rating of the particular segment, which was collected after the test. White color (1) is lowest stress; black color (6) is highest stress.

The Skin conductance timeline shows values of the GSR, which are scaled into the height of the line view. The exact values are shown in the Detail window (described in following section).

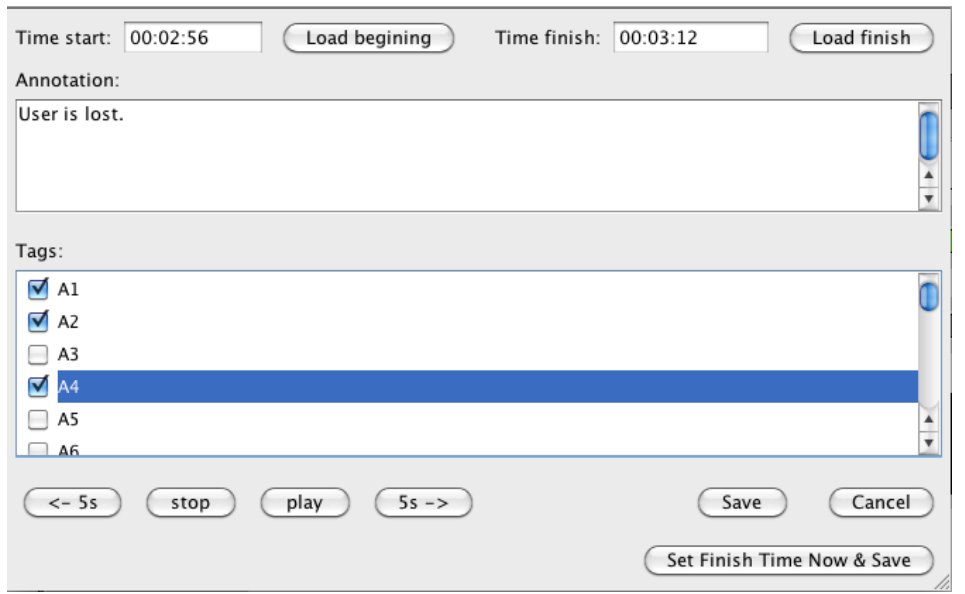

Fig 7 Annotation window detail

The Annotations timeline shows additional annotations added during the analysis of the data in the IVE tool. For each annotation there is a rectangle that represents start and end time of the annotation. Annotation details are shown in the Detail window. Each annotation (see detail of annotation editor in Fig 7) can store annotation description and a set of tags that may be used for filtering of the annotations. There are also basic video controls for easier setting of start and end times and for repeating observation of a particular point.

Apart from the data analysis the Timeline visualization plug-in is used also for time synchronization of timeline data sets with video. All the data sets in the Timeline visualization plug-in use time of the day so we can easily transform this time into the time of the master video. For this purpose we use the Timeline video synchronization plug-in (see Fig 8). We find the same time point in the Timeline visualization plug-in view and in the Video plug-in and add it to the Video log synchronizer window and synchronize. 


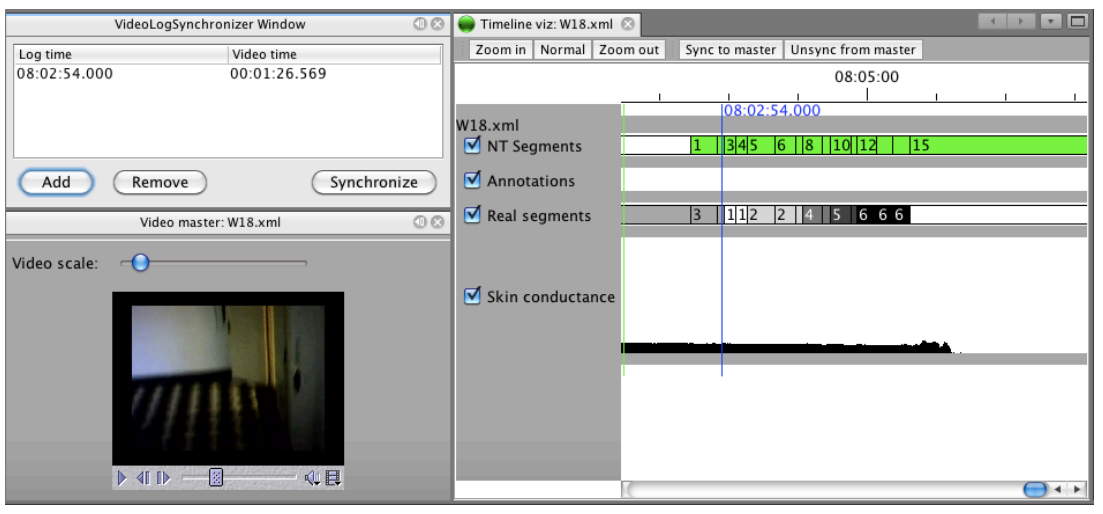

Fig 8 Video log synchronization plug-in

\subsection{Multiple video visualization}

The Video viewer plug-ins are the plug-ins for sequential replay of all video files. One video viewer plug-in always acts as a master synchronization point, i.e. all other plug-ins synchronize to the master time. In case we have more then one video source, we can use other video viewer plug-ins which are then also synchronized to the master video viewer plug-in. The Video viewer plug-in contains a scale slider which allows adjustment of the video depending on the location in the IVE tool. The Slave video viewer plug-in also contains buttons for synchronization with the master video viewer plug-in (see Fig 9). During the test's data analysis we used one master view. The number of slave views is limited by the resources of the PC and operating system.

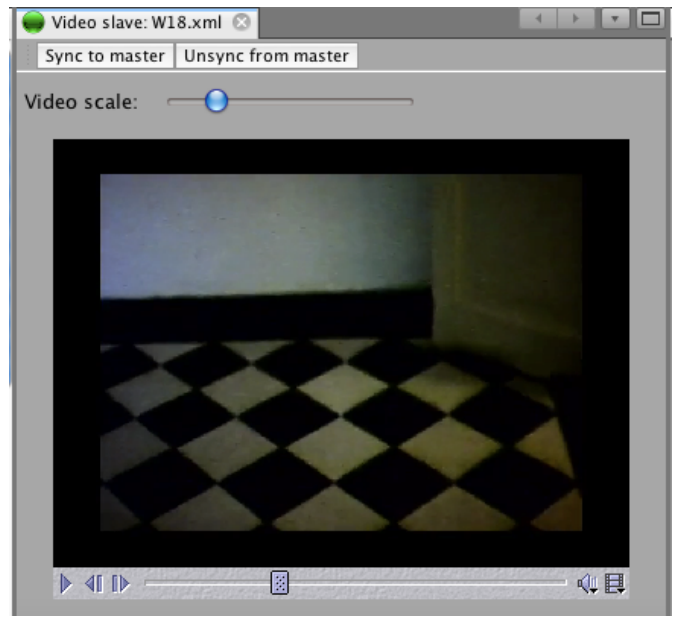

Fig 9 Slave video viewer plug-in

\subsection{Segment Visualization Plug-in}

Segments are visualized mainly in the timeline. However, there is additional visualization of the route segment area in a form of image (see Fig 10). The segment visualization is synchronized with the master video and with the real segment data source to show the current segment. There is also a scale slider, which allows for adjustment of the plug-in view depending on the location in the IVE tool. 


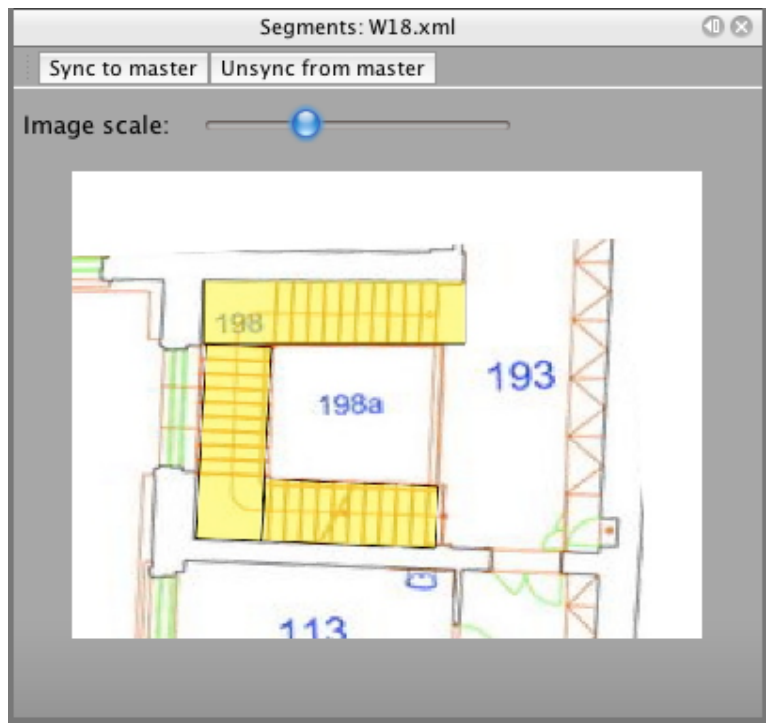

Fig 10 Route segment visualization plug-in view

\subsection{Heart Rate Variability parameter visualization}

As the HRV parameter analysis is based on the FFT, the obtained values are not assigned to a certain time. In the IVE tool, the Heart Rate Variability (HRV) visualization plug-in shows HRV data sets in form of a bar graph (see Fig 11). The first bar represents the LF/HF ratio for the first 4 minutes (4 minute sliding window of FFT) of the analyzed signal. The second bar represents the LF/HF ratio for signal from time 1:00 min to 5:00 min. Every other bar represents sliding the window by 1 minute.

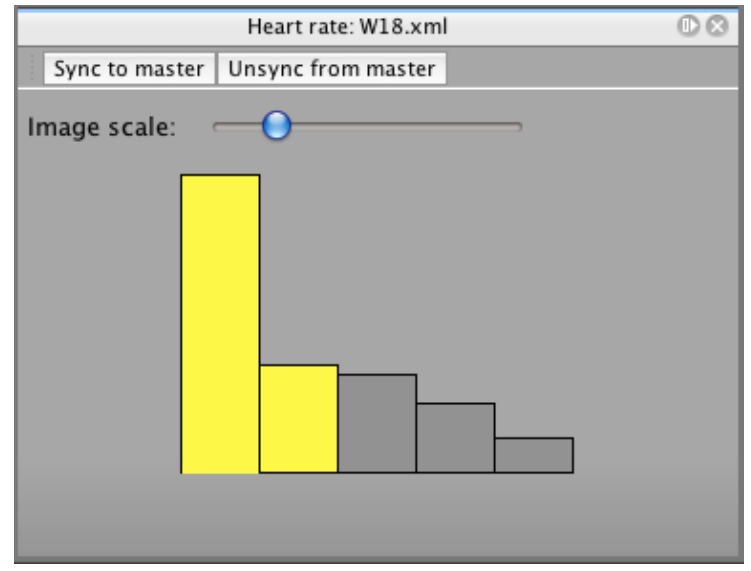

Fig $11 \mathrm{HRV}$ data visualization plug-in view

Exact LF/HF ratio value is shown in Detail window. The color of the bar is yellow when the actual time of video playback in the Video viewer plug-in intersects the corresponding sliding window interval. Therefore up to 4 bars can be indicated as "active" and highlighted in yellow. 


\subsection{Detail window}

The Detail window is a multipurpose window for visualization of plug-in record details. In the window we show a list of key value pairs that are sent by the particular visualization plug-in. In Fig 12 you can see detail information for the segment. There are navigation instructions derived from the application model data set. There are also interaction points (Points) that the participant interacted with during the test and that the participant mentioned in the after test interview.

\begin{tabular}{|c|c|}
\hline & DetailObserver Window \\
\hline Key & Value \\
\hline segment & 4 \\
\hline popis & $\begin{array}{l}\text { DESCRIPTION Corridor, about } 8 \text { meters } \\
\text { long. On the right there is door to toilete. } \\
\text { The corridor turns right at the end. } \\
\text { Before the end of the corridor, there is } \\
\text { extinguisher on the right. }\end{array}$ \\
\hline nextSegment & 5 \\
\hline lod & 0 \\
\hline state & Segment 4 \\
\hline class & real_segment \\
\hline transitionType & 0 \\
\hline id & 400008 \\
\hline akce & $\begin{array}{l}\text { ACTION Go to the end of the corridor } \\
\text { and turn right. }\end{array}$ \\
\hline time & 08:03:16.000-01.01.1970 \\
\hline edge_id & 143 \\
\hline command & Dalsi \\
\hline points & $\begin{array}{l}\text { In test and extern: } \\
\text { In test: } \\
\text { In extern: } \\
\text { Not metioned: } \\
\text { A8 - door (WC) } \\
\text { A9 - cabinet } \\
\text { A10 - embrasure } \\
\text { A11 - extinguisher }\end{array}$ \\
\hline
\end{tabular}

Fig 12 Detail window

\subsection{Performed analysis}

In the test we collected data from 22 participants. The length of the tests was between 8 and 30 minutes. For each participant we got a log file from the application, his/her GSR data (from approx. 6000 to 19000 records), HRV data (from 4 to 20 sliding window values), log sheets from the test and from the posttest route description. For each participant we recorded 2 videos.

The time for data synchronization was a maximum of 5 minutes and the speed of annotation corresponded with the findings from our previous studies with the IVE tool [3].

\section{Conclusion}

In this paper we have demonstrated tools and methods for efficient evaluation of experiments in mobile and realistic environments. Our demonstration use-case was the "Navigation of visually impaired person inside buildings". During such experiments a much larger amount of data of various kinds is generated in comparison with static and lab based experiments. We have shown that for these kinds of experiments other methods for processing, visualization and analysis 
should be used. More specific, our analytical tool IVE is capable of handling huge amounts of data of very different types. It offers advanced features for synchronization of data (e.g., synchronization of audio/visual data with HRV data that does not have exact time assignment), visualization and analysis of contextual data like application state, graphical representation of the participant's surrounding, and interaction of the participant with objects on the route.

Thanks to the IVE tool we were able to analyze the stress measurement data in the context of uncontrolled situational variables that potentially cause stress and distinguished them from a true indication of stress. These data were omitted from further analysis. Without the annotation plug-ins and advanced synchronization features of the IVE tool we would be unable to detect the false indicators. Through the global view on the various data provided by the IVE tool we were also able to detect other stress stimuli that were hidden to us from simple watching of videos (e.g., GSR values were higher when the user had to concentrate enormously on the NaviTerier route description).

During the experiment preparation and evaluation we found that we need some very special plug-ins (e.g., visualization of participant surroundings during route walkthrough). Thus we appreciated the efficient plug-in interface of the IVE tool, which in less than 3 weeks allowed us to develop 5 new plug-ins (described in section 6).

Although the IVE tool was evaluated as useful there are still some areas where we want to focus in future work. We found that applications with a complex layout of windows, like IVE, are quite difficult to set up and there should be assistance in the set up and saving of the window layout. Also there should be easier switching between the data set visualization, e.g. switching between 2 users. Currently we have to reload all the visualization plug-in views. From the test point of view we want to focus on analysis of interactions between the user and other objects during the test, e.g. visualize such interaction in a segment view plug-in.

\section{Acknowledgement}

This research has been partially supported by the MSMT under the research program MSM 6840770014. This research has also been partially supported by the MSMT under the research program LC-06008 (Center for Computer Graphics).

We would like to thank P. Smrcka and R. Kliment from the Joint Department of Biomedical Engineering CTU and Charles University in Prague, Studnickova 7/2028 Praha 2 for consultation and lending of the ECG for measuring HW and SW.

We would like to thank the BioDat research group, Gerstner Laboratory, Department of Cybernetics, Faculty of Electrical Engineering, Czech Technical University in Prague for lending of the GSR measuring device. 


\section{References}

[1] Sandi C, Pinelo-Nava MT (2007) Stress and memory: behavioral effects and neurobiological mechanisms. Neural Plast 2007 2007:78970. doi:10.1155/2007/78970

[2] Salahuddin L, Desok K (2011) Detection of Acute Stress by Heart Rate Variability Using a Prototype Mobile ECG Sensor. http://vega.icu.ac.kr/ kimdesok/IEEE-CS_IEEE251.pdf. Accessed on 12 February 2011

[3] Maly I, Mikovec Z, Vystrcil J (2010) Interactive Analytical Tool for Usability Analysis of Mobile Indoor Navigation Application. In: Proceedings of the 3rd International Conference on Human System Interaction (HSI 2010), pp. 259-266, doi: 10.1109/HSI.2010.5514559

[4] Mackinlay JD (1999) Readings in information visualization: using vision to think. Card, S.K., Shneiderman, B. (eds.), Morgan Kaufmann Publishers Inc., San Francisco, pp. 686

[5] Kubios HRV (2011) University of Eastern Finland, http://kubios.uku.fi/, Biosignal Analysis and Medical Imaging Group. Accessed 10 April 2010.

[6] Kjeldskov J, Skov MB, Als BS et al. (2004) Is it Worth the Hassle? Exploring the Added Value of Evaluating the Usability of Context-Aware Mobile Systems in the Field. In: Proceedings of the MobileHCl conference 2004, pp 61-73, doi: 10.1007/978-3-54028637-0_6

[7] Visifire (2011) http://www.visifire.com/. Accessed 1 February 2011

[8] Noldus (2011) Human behavior research. http://www.noldus.com/human-behaviorresearch. Accessed 26 January 2011

[9] Kitchin R, Jacobson RD (1997) Techniques to collect and analyze the cognitive map knowledge of persons with visual impairment or blindness: Issues of validity. J Vis Impair Blind 91:360-376

[10] Bayevsky RM, Ivanova GG, Chireykin LV, Gavrilushkin AP, Dovgalevsky PYa, Kukushkin UA, Mironova TF, Priluzkiy DA, Semenov UN, Fedorov VF, Fleishmann AN, Medvedev MM (2002) HRV Analysis under the usage of different electrocardiography systems (Methodical recommendations). These methodical recommendations are prepared according to the order of the Committee of Clinic Diagnostic Apparatus and the Committee of New Medical Techniques of Ministry of Health of Russia (protocol 4 from the 11th of April, 2002), Moskva, 2002, http://www.vestart.ru/atts/1267/24baevsky.pdf. Accessed 1 February 2011.

[11] Benoit A, Bonnaud L (2009) Multimodal focus attention and stress detection and feedback in an augmented driver simulator. Pers Ubiquitous Comput 13(1):33-41. doi: 10.1007/s00779-007-0173-0

[12] Gray JA (1987) The psychology of fear and stress. Cambridge University Press, Cambridge

[13] Foo P, Warren WH, Duchon A, Tarr MJ (2005) Do humans integrate routes into a cognitive map\&quest; Map versus landmark-based navigation of novel shortcuts. J Exper Psych Learn Memory Cognition 31:195-215 
[14] Bassett JR, Marshall PM, Spillane R (1987) The physiological measurement of acute stress (public speaking) in bank employees. Int J Psychophysiol 5(4):265-273. doi: 10.1016/0167-8760(87)90058-4

[15] Yamanaka K, Kawakami M (2011) Convenient Evaluation of Mental Stress With Pupil Diameter. http://www.ciop.pl/33712 Accessed 1 February 2011.

[16] Ohsuga M, Shimono F, Genno H (2001) Assessment of physical work stress using autonomic indices. Int J Psychophysiol 40:211-220. doi: 10.1016/S0167-8760(00)001896

[17] Berntson GG, Bigger JT, Eckberg DL, et al. (1997) Heart rate variability: origins, methods, and interpretive caveats. Psychophysiology 34(6):623-648. doi: 10.1111/j.14698986.1997.tb02140.x

[18] Hána K, Smrčka P, Kašpar J, Fiala R, Mužík J, et al. (2009) The system for monitoring of the human psychophysiological state utility model No. 19422, Czech Industrial Property Office

[19] Gillner S, Mallot HA (1998) Navigation and acquisition of spatial knowledge in a virtual maze. J Cogn Neurosci 10:445-463. doi: 10.1162/089892998562861

[20] Tellevik JM (1992) Influence of spatial exploration patterns on cognitive mapping by blindfolded sighted persons. J Vis Impair \& Blind 86:221-224

[21] Ochaíta E, Huertas JA (1993) Spatial representation by persons who are blind: a study of the effects of learning and development. J Vis Impair \& Blind 87:37-41 